\title{
AUTOMATIZACIÓN DEL PROCESO DE TOMA DE INVENTARIOS CÍCLICOS EN UNA EMPRESA COMERCIAL UBICADA EN LIMA Y SU EFECTO EN LA REDUCCIÓN DE GASTOS
}

\section{Automation of the Process of Taking Cyclic Inventories in a Commercial Company Located in Lima and its Effect on the Reduction of Expenses}

\author{
Víctor Hugo Fernández Bedoya, MsC. \\ Universidad César Vallejo, Perú \\ https://orcid.org/0000-0002-2464-6477 \\ victorhugofernandezbedoya@gmail.com
}

Palabras claves: Toma de Inventarios Cíclicos, Automatización, Reducción de Gastos, Empresas Comerciales.

Keywords: Cyclical Inventories, Automation, Cost Reduction, Commercial Companies.
Recibido: 18 de diciembre de 2019

Aceptado: 06 de marzo de 2020

\section{RESUMEN}

Esta investigación describe y a la vez analiza una iniciativa de innovación llevado a cabo en una empresa comercial ubicada en Lima a inicios del año 2013, donde la automatización del proceso de toma de inventarios cíclicos implementada disminuyó los gastos de ventas de dicha compañía, los cuáles son significativos y cuya reducción redundó directamente en el incremento en las utilidades netas de la empresa comercial estudiada. La metodología utilizada consta de un cuestionario que contiene 14 preguntas a manera de encuesta que permitieron conocer el perfil y conocimientos de los jefes de ventas encargados de la realización de conteos cíclicos, y también de su conocimiento y expectativas en relación a los gastos de ventas relacionados a esta actividad; además, se muestra análisis de resultados financieros, donde se demuestra que la automatización de la toma de inventarios a través del uso de scanners redujo los gastos de ventas en S/ 1 886,50 mensuales (S/ 22 638,00 anuales), y que, de replicarse esta innovación en el resto de sucursales de esta cadena de tiendas, se lograría reducir los estos gastos en S/ 205 800,00 , lo cual representa una reducción del 0,05\% por concepto de ventas del año anterior.

\begin{abstract}
This research describes and at the same time analyzes an innovation initiative carried out in a commercial company located in Lima at the beginning of 2013, where the automation of the process of taking cyclical inventories implemented reduced the sales expenses of the company, which are significant and whose reduction directly resulted in the increase in net profits of the commercial company studied. The methodology consists of a questionnaire that contains 14 questions as a survey that allowed to know the profile and knowledge of the sales managers in charge of making cyclical inventories, and also to their knowledge and expectations in relation to sales expenses related to this activity; in addition, an analysis of financial results is shown, which shows that the automation of inventory taking through the use of scanners reduced sales expenses by S/ 1886.50 per month ( $\mathrm{S} / 22638.00$ per year), and that if this innovation were replicated in the rest of the branches of this chain of stores, they would be able to reduce these expenses by S/ 205800.00 , which represents a reduction of $0.05 \%$ from the sales of the previous year.
\end{abstract}




\section{INTRODUCCIÓN}

En toda empresa de actividad de venta al por menor es imprescindible la realización de inventarios cíclicos (frecuentes y programados), de esta manera se prevén y corrigen deficiencias en relación a la disponibilidad de mercadería (Agüero Zardón, Urquiola Garcia y Martínez Delgado, 2016; Durán, 2017; Garrido Bayas y Cejas Martínez, 2017; Gutiérrez y Vidal, 2008), esto debido a que la información mostrada en los inventarios podría no ser exacta debido a diversos motivos (faltantes de mercadería al momento de la recepción, ingreso físico de productos no listados en la guía de recepción, siniestros, etc.).

El sistema de inventarios cíclicos consiste en la implementación de un sistema efectivo de control de pérdidas a través de la toma de inventarios de los grupos de productos que generan el mayor acortamiento (pérdida) para una tienda.

La empresa en la cual se desarrolló la investigación selecciona los productos a los cuales se realizarán los conteos cíclicos mediante el análisis $A B C$, el cual se basa en la regla 80-20 o Ley de Pareto y es considerado el método más sofisticado en la actualidad (Olivos Aarón y Penagos Vargas, 2013).

El proceso de toma de inventario cíclico que se lleva a cabo en la sucursal de San Isidro de esta empresa comercial se realiza manualmente, siguiendo generalmente el siguiente proceso:

a) El jefe de control de existencias de la tienda genera el reporte de stock disponible del tipo de producto al que se realizará inventario cíclico (ejemplo: todas las zapatillas de dama, marca Adidas) y lo remite al jefe de ventas vía correo electrónico.

b) El jefe de ventas recibe el correo electrónico, e imprime el listado de productos a contar, siendo necesarias en total 3 copias.

c) El personal encargado de conteo cíclico recoge el listado de productos a contar, y realizan el conteo cíclico de manera manual, identificando el código de la prenda, y buscándolo en el listado previamente impreso, de entre varios otros códigos. Cabe resaltar que este grupo encargado de conteo está conformado usualmente por tres vendedores y el jefe de ventas, supervisados por un representante del área de seguridad. El conteo cíclico dura 1 hora y 45 minutos, realizándose entre las 9:15 a.m. y 11:00 a.m. (antes de que la tienda abra al público), generando horas extras para el personal que asiste al conteo cíclico.

d) Terminado el conteo cíclico, el jefe de ventas remite información del conteo vía mail al jefe de control de existencias.

e) El jefe de control de existencias realiza análisis de diferencias y emite un reporte y recomendaciones.

La realidad problemática que se suscitó en esta actividad es que este proceso, manual, consume demasiados recursos, generando horas extras a personal, además de un uso excesivo de impresiones, lo cual se traduce, financieramente en gastos de ventas que minimizarán las utilidades de la compañía; cabe recordar que el objetivo primordial de toda empresa con fines de lucro es, precisamente, la generación de ganancias (Fernández Bedoya, 2018).

Se estimó que el gasto incurrido diariamente en el conteo cíclico tradicional era de S/ 105,95 en horas extras y en la impresión de hojas bond, como se realizaban en frecuencia de 5 días a la semana, en 1 mes (20 días de conteo) el gasto de ventas total era de $S / 105,95 * 20=S / 2119,00$

Por primera vez este estudio demostró cuánto afectaba a los gastos de venta el pago de horas extras producto de los conteos cíclicos, por lo que se tomó la iniciativa de buscar alternativas para reducir estas horas extras; se estaba poniendo en práctica lo recalcado siempre por las gerencias en los más altos niveles de la empresa cuando se dictan charlas y conferencias a los colaboradores de la empresa : fomentar, cumplir, con la puesta en práctica de los valores de la austeridad y de la toma de iniciativas que son considerados valores fundamentales que soportan la visión de la empresa. El buen rendimiento de cualquier empresa generalmente es debido al éxito de las estrategias que se implementen (Esteves Pairazamán y Fernández Bedoya, 2019).

Gracias a la política de transparencia que practica la empresa, el jefe de control de existencias logró conseguir 12 mini scanner ópticos marca Symbol modelo CS1504, los cuáles debido a su antigüedad ya estaban totalmente depreciados y eran considerados obsoletos para los fines que fueron inicialmente adquiridos (programa de lista de novios), estos fueron rápidamente reconfigurados para que fueran utilizados en actividades de conteo cíclico de mercaderías, haciendo de esta manera el trabajo más ligero, requiriendo para ello menos personal y tiempo. 


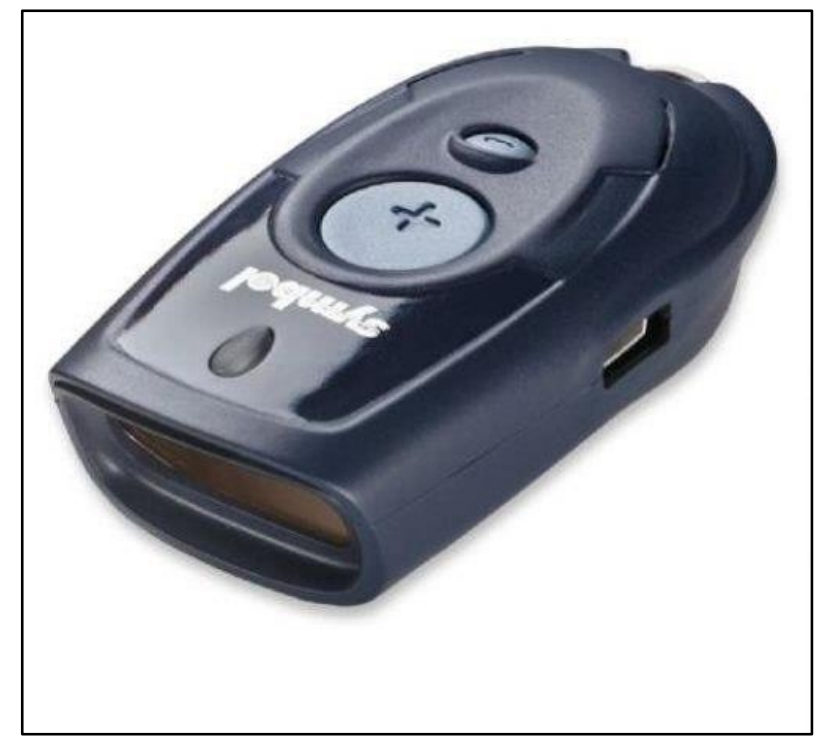

Figura 1: Mini scanner óptico marca Symbol, modelo CS1504

El principal problema con el conteo cíclico tradicional, adicional a sus gastos de ventas ya mencionados, era la gran cantidad de códigos con que se identifican los productos que vende esta empresa comercial y que al momento de registrarlo en los listados, los vendedores demoraban en ubicar la fila en la que estaba impreso el código (de doce dígitos), esta actividad a su vez generaba fatiga visual; en cambio, utilizando scanners no se genera fatiga visual porque tan solo es necesario presionar el botón, y la información del código queda registrada en el escáner que cuenta con un gran poder de almacenamiento de información gracias a la TIC.

Cabe precisar que los scanners son aparatos pequeños que permiten registrar el código de barras de los productos y pueden ser utilizados en los conteos cíclicos debido a la memoria interna que poseen; el scanner de códigos de barras a menudo produce tasas de exactitud de datos de hasta 99 \% (Glover y Bhatt, 2009) esta tecnología supera por mucho a la entrada de datos manual (papel y lapicero), además de ser más rápido y no generar fatiga visual.

\section{DESARROLLO}

Esta investigación es de modalidad factible, debido a que consiste en la investigación, elaboración, desarrollo de una propuesta de un modelo operativo viable para solucionar problemas, requerimientos o necesidades de organizaciones (Dubs de Moya, 2012; Sanca Tinta, 2011).

Cabe resaltar, que para esta investigación se realizó una encuesta a una muestra de 16 jefes de ventas, quienes son los responsables de la correcta ejecución de los conteos cíclicos, para conocer su perfil, y su conocimiento y expectativas en relación a la toma de inventarios cíclicos utilizando scanners. Las encuestas fueron realizadas de manera presencial y los resultados tabulados y graficados utilizando Microsoft Excel.

En cuanto el análisis de los resultados financieros de la compañía, se compararon los gastos incurridos durante un mes de toma de inventarios cíclicos utilizando el método tradicional (manual), y los gastos incurridos durante la toma de inventarios cíclicos de los mismos productos utilizando el método propuesto durante el mes siguiente. A partir de los datos obtenidos se determinó a cuánto asciende el ahorro en gastos de ventas diario, mensual, y anual, y a cuánto ascendería en caso se aplique este ejercicio en todas las sucursales de la compañía.

A continuación, se presentarán los resultados tanto de la encuesta como del análisis de los resultados financieros.

\section{Análisis de los resultados de la encuesta}

La encuesta constó de 14 preguntas, las 7 primeras con nos muestran el perfil y conocimientos del encuestado, las 7 siguientes nos dan a conocer el conocimiento y expectativas del encuestado en relación a los gastos del conteo cíclico. 
a) Perfil y conocimientos del encuestado:

\begin{tabular}{|c|c|c|c|}
\hline Pregunta & Atributos & Valor absoluto & Valor relativo \\
\hline 1 & $\begin{array}{l}\text { Tipo de producto del cual el encuestado } \\
\text { está a cargo. (Calzado, accesorios y/o } \\
\text { textiles). }\end{array}$ & 10 & $63 \%$ \\
\hline 2 & $\begin{array}{l}\text { Experiencia dentro de la empresa. ( } 5 \text { a } \\
\text { más años). }\end{array}$ & 10 & $63 \%$ \\
\hline 3 & $\begin{array}{l}\text { Considera que es muy importante la } \\
\text { realización de inventarios cíclicos. }\end{array}$ & 16 & $100 \%$ \\
\hline 4 & $\begin{array}{l}\text { Conoce de la existencia de scanners para } \\
\text { la realización de inventarios cíclicos. }\end{array}$ & 16 & $100 \%$ \\
\hline 5 & $\begin{array}{l}\text { Considera que está poco informado sobre } \\
\text { el uso de los scanners para la realización } \\
\text { de inventarios cíclicos. }\end{array}$ & 13 & $81 \%$ \\
\hline 6 & $\begin{array}{l}\text { Está muy de acuerdo en realizar } \\
\text { inventarios cíclicos utilizando scanners. }\end{array}$ & 13 & $81 \%$ \\
\hline 7 & $\begin{array}{l}\text { Está muy de acuerdo en ser capacitado } \\
\text { en el uso de scanners para la realización } \\
\text { de inventarios cíclicos con los mismos. }\end{array}$ & 16 & $100 \%$ \\
\hline
\end{tabular}

Tabla 1: Valores absolutos y relativos de las 7 preguntas referentes al perfil y conocimientos del encuestado

De acuerdo con la tabla 1, la investigación se realizó a los 16 jefes de ventas que están a cargo de diferentes áreas dentro de la tienda analizada, pudiéndose observar que el $63 \%$ de ellos cuentan con más de 5 años laborando en la compañía.

Se notó que el $63 \%$ de ellos está a cargo de áreas donde se venden productos de calzado, accesorios y/o textiles, los cuales son líneas de productos de gran susceptibilidad al robo, por lo que necesitan estar conscientes y actualizar minuciosamente su stock de mercadería, es por eso que todos (100\%) consideran muy importante la realización de inventarios cíclicos.

En cuanto a sus conocimientos sobre los scanners para la realización de tomas de inventarios cíclicos, se pudo conocer que todos (100\%) conoce de su existencia, esto es debido al trato directo que tienen con proveedores de productos que no pertenecen a la tienda, por experiencias laborales previas, ó por simple cultura general.

Sin embargo, la gran mayoría ( $81 \%$ ) considera que está poco informado sobre su correcto uso y sus beneficios; es por ello que luego de explicarles las principales características y beneficios de los scanners, el 81 \% respondió estar muy 
de acuerdo en su uso; asimismo, se mostraron interesados en este nuevo método, prueba de ello es que el $100 \%$ de los encuestados respondió estar muy de acuerdo en ser capacitado en el correcto y eficaz uso de estos scanners y sus beneficios de este último.

b) Conocimiento y expectativas del encuestado en relación a los gastos del conteo cíclico:

\begin{tabular}{|c|c|c|c|}
\hline Pregunta & Atributos & Valor absoluto & Valor relativo \\
\hline 8 & $\begin{array}{c}\text { Nunca realiza conteos cíclicos dentro de } \\
\text { las horas de trabajo, porque le generan } \\
\text { horas extras. }\end{array}$ & 16 & $100 \%$ \\
\hline 9 & $\begin{array}{c}\text { Está muy de acuerdo en que los } \\
\text { inventarios sean realizados por } 1 \\
\text { persona, en vez de } 4 .\end{array}$ & 16 & $100 \%$ \\
\hline 10 & $\begin{array}{c}\text { Desearía que se reduzcan mucho los } \\
\text { gastos de la compañía, en relación a } \\
\text { pagos por horas extras. }\end{array}$ & 16 & $100 \%$ \\
\hline 11 & $\begin{array}{c}\text { Considera que si utiliza scanners para la } \\
\text { toma de inventarios cíclicos se reducirá } \\
\text { mucho los errores de conteo. }\end{array}$ & 14 & $88 \%$ \\
\hline 12 & $\begin{array}{c}\text { Considera que si utiliza scanners para la } \\
\text { toma de inventarios cíclicos los realizará } \\
\text { más rápido. }\end{array}$ & 13 & $81 \%$ \\
\hline 13 & $\begin{array}{c}\text { Considera que si utiliza scanners para la } \\
\text { toma de inventarios cíclicos se reducirá } \\
\text { mucho los gastos empresariales. }\end{array}$ & 13 & $81 \%$ \\
\hline 14 & $\begin{array}{c}\text { Considera que si utiliza scanners para la } \\
\text { toma de inventarios cíclicos se } \\
\text { incrementará mucho la rentabilidad de la } \\
\text { compañía. }\end{array}$ & 13 & $81 \%$ \\
\hline
\end{tabular}

Tabla 2: Valores absolutos y relativos de las 7 preguntas referentes al conocimiento y expectativas del encuestado en relación a los gastos del conteo cíclico.

Como se observa en la tabla 2 , el $100 \%$ de los encuestados, y sus grupos, nunca realizan conteos cíclicos con su equipo dentro de las horas de trabajo, por lo que generan horas extras y son conscientes de ello; además, el $100 \%$ está muy de acuerdo en que estos conteos cíclicos sean realizados por 1 sola persona en lugar de 4.

Con respecto a los gastos de la compañía, el $100 \%$ de los encuestados coinciden en que desearían mucho reducirlos sustancialmente.

Del total de los encuestados, 14 (88 \%) considera que si utilizamos scanners para la toma de inventarios cíclicos reduciremos mucho los errores de conteo; a su vez el $75 \%$ responde que si utilizamos scanners para la toma de inventarios cíclicos los realizaremos mucho más rápido.

El $81 \%$ de los encuestados considera que si utilizamos scanners para la toma de inventarios cíclicos reduciremos mucho los gastos empresariales, esta misma proporción de encuestados también considera que si utilizamos scanners para la toma de inventarios cíclicos se incrementará mucho la rentabilidad de la compañía.

\section{Análisis de los resultados financieros}

A continuación, se contrastará a través de gráficos relevantes los resultados en cuanto a los gastos de ventas incurridos con los dos métodos de toma de inventario cíclico: tradicional, expuesto en la tabla 3; y automatizado (método propuesto), expuesto en la tabla 4. 


\begin{tabular}{|c|c|c|c|c|}
\hline $\begin{array}{l}\text { Cargo del } \\
\text { personal }\end{array}$ & $\begin{array}{c}\text { Cantidad de } \\
\text { personal }\end{array}$ & $\begin{array}{l}\text { Tarifa por } \\
\text { hora extra }\end{array}$ & $\begin{array}{c}\text { horas extra } \\
\text { generadas por } \\
\text { cada persona }\end{array}$ & Total gasto \\
\hline Vendedor & 9 & $S / 3,75$ & $13 / 4$ & $S / 59,06$ \\
\hline J. Ventas & 3 & $S / 7.00$ & $13 / 4$ & S/ 36.,75 \\
\hline Seguridad & 1 & $\mathrm{~S} / 4,25$ & $13 / 4$ & $S / 7,44$ \\
\hline \multicolumn{4}{|c|}{ Total gastos de ventas (recursos humanos) } & $S / 103,25$ \\
\hline $\begin{array}{l}\text { Otros } \\
\text { recursos }\end{array}$ & $\begin{array}{l}\text { Cantidad de } \\
\text { personal }\end{array}$ & $\begin{array}{l}\text { Costo por } \\
\text { recurso }\end{array}$ & $\begin{array}{l}\text { Cantidad de } \\
\text { recursos } \\
\text { utilizados por } \\
\text { persona }\end{array}$ & Total gasto \\
\hline $\begin{array}{l}\text { Hojas de } \\
\text { conteo }\end{array}$ & 9 & $S / 0.10$ & 2 & $S / 1,80$ \\
\hline $\begin{array}{l}\text { Hojas de } \\
\text { reconteo }\end{array}$ & 3 & $S / 0.10$ & 3 & $S / 0,90$ \\
\hline \multicolumn{4}{|c|}{ Total gastos de ventas (recursos materiales) } & $\mathrm{S} / 2,70$ \\
\hline \multicolumn{4}{|c|}{ Total gastos de ventas (conteo manual) } & $S / 105.95$ \\
\hline
\end{tabular}

Tabla 3: Gastos incurridos durante 01 día de toma de inventarios cíclicos de manera manual

\begin{tabular}{|c|c|c|c|c|}
\hline $\begin{array}{l}\text { Cargo del } \\
\text { personal }\end{array}$ & $\begin{array}{l}\text { Cantidad de } \\
\text { personal }\end{array}$ & $\begin{array}{l}\text { Tarifa por hora } \\
\text { extra }\end{array}$ & $\begin{array}{c}\text { horas extra } \\
\text { generadas por } \\
\text { cada persona }\end{array}$ & Total gasto \\
\hline Vendedor & 3 & $\mathrm{~S} / 3,75$ & $3 / 4$ & $\mathrm{~S} / 8,44$ \\
\hline Seguridad & 1 & S/ 4,25 & $3 / 4$ & $S / 3,19$ \\
\hline \multicolumn{4}{|c|}{ Total gastos de ventas (recursos humanos) } & $\mathrm{S} / 11,63$ \\
\hline $\begin{array}{l}\text { Otros } \\
\text { recursos }\end{array}$ & $\begin{array}{l}\text { Cantidad de } \\
\text { personal }\end{array}$ & $\begin{array}{l}\text { Costo por } \\
\text { recurso }\end{array}$ & $\begin{array}{l}\text { Cantidad de } \\
\text { recursos } \\
\text { utilizados por } \\
\text { persona }\end{array}$ & Total gasto \\
\hline $\begin{array}{l}\text { Hojas de } \\
\text { conteo }\end{array}$ & 3 & $S / 0.10$ & 0 & $\mathrm{~S} / 0,0$ \\
\hline \multicolumn{4}{|c|}{ Total gastos de ventas (recursos materiales) } & $S / 0,00$ \\
\hline \multicolumn{4}{|c|}{ Total gastos de ventas (conteo automatizado) } & $S / 11.63$ \\
\hline
\end{tabular}

Tabla 4: Gastos incurridos durante 01 día de toma de inventarios cíclicos de manera automatizada

Previo al proceso de innovación presentado, la ejecución del sistema de inventarios cíclicos utilizaba el método tradicional que consistía en imprimir los listados de los productos a contar y distribuirlos entre el personal de conteo, requiriendo para ello 3 vendedores y 1 jefe de ventas para la ejecución del conteo cíclico de cada área; como cada día se tiene que realizar el conteo cíclico de 3 áreas, diariamente se requería de 9 vendedores y de 3 Jefes de Ventas, además de un personal de seguridad para validar los conteos.

Estos conteos realizados de lunes a viernes ( 5 días a la semana y 20 días al mes) demoraban 1 hora y 45 minutos, y se realizaban fuera de las horas de trabajo; durante la ejecución del presente trabajo de investigación se estimó que los gastos incurridos diariamente en el conteo cíclico tradicional era de S/ 105,95 (n horas extras y en la impresión de hojas bond (ver detalle en figura 4); como se trabajaba 5 días a la semana, en 1 mes (20 días de conteo) el gasto total era de $\mathrm{S} / 105,95 \times 20=\mathrm{S} / 2119,00$. 
A partir de la iniciativa propuesta por el jefe de control de existencias, los conteos cíclicos se realizaron con el apoyo de scanners; asimismo, se continúan realizando 3 conteos al día, pero sólo es necesario que 1 persona realice el conteo cíclico debido a que se utilizan scanners; estos conteos ahora duran 45 minutos pagándose tan solo S/ 11,63 por concepto de horas extras por cada día en que se ejecutan los conteos cíclicos (ver figura 5); en 1 mes (20 días de conteo) se paga $\mathrm{S} / 232,50$ en lugar de los $\mathrm{S} / 2119,00$ que se gastaba anteriormente; esto significaba un ahorro ascendiente a $\mathrm{S} / 2119,00-\mathrm{S} / 232,50=\mathrm{S} / 1886,50$ mensuales y de $\mathrm{S} / 1886,50 \times 12=\mathrm{S} / 22$ 638,00 anuales en los gastos de ventas en la empresa en tan sólo la sucursal ubicada en San Isidro; asimismo, como los gastos de ventas son importantes dentro de la estructura de costos de la empresa, cualquier reducción de dichos gastos facilitará la obtención de mayores utilidades netas de esta empresa.

De otro lado, teniendo en cuenta que la donde se realizó está innovación representa el $11 \%$ del total de las ventas de la cadena de tiendas de la compañía, entonces, si se considera que las 22 sucursales de la empresa adoptasen la innovación de los scanners en sus respectivos conteos cíclicos, el ahorro total anual en gastos de ventas sería de S/ $22638,00 / 11 \%=S / 205800,00$ que es una suma respetable porque representa el $(205800 / 430900000) \times 100=$ $0,05 \%$ del total de gastos de ventas correspondiente al año 2012; asimismo, este ahorro anual en gastos de ventas representa el $(205800 / 129100000) \times 100=0,17 \%$ del total de la utilidad neta de la empresa durante el año 2012 (ver tablas 5 y 6 ).

\section{Ventas netas de la cadena y de la tienda San Isidro}

\begin{tabular}{cccc} 
& & \multicolumn{2}{c}{ millones soles } \\
\cline { 3 - 4 } A & AÑO $\rightarrow$ & 2011 & 2012 \\
B & Total Cadena & 2180 & 2422 \\
& Tienda San Isidro & 237 & 256 \\
B/A en \% & $11 \%$ & $11 \%$
\end{tabular}

Tabla 5: Ventas netas de la cadena y de la tienda ubicada en San Isidro, Lima.

Estado de ganancias y pérdidas de la cadena de tiendas

Expresado en millones de soles

\begin{tabular}{lrrrr}
\hline \hline & 2009 & 2010 & 2011 & 2012 \\
Ventas & 1624,0 & 1847,6 & 2180,3 & 2422,3 \\
costo de ventas & 1165,4 & 1268,0 & 1527,1 & 1717,1 \\
utilidad bruta & 458,6 & 579,6 & 653,2 & 705,2 \\
Gastos & 314,5 & 363,0 & 464,2 & 519,9 \\
\hline gasto de ventas & 252,6 & 290,5 & 374,7 & 430,9 \\
\hline gasto de administración & 61,9 & 72,5 & 89,5 & 89,0 \\
utilidad de operación & 144,1 & 216,6 & 189,0 & 185,3 \\
Gastos (e ingresos) & 1,5 & 20,8 & 19,5 & 8,2 \\
financieros. & 145,6 & 237,4 & 208,5 & 193,5 \\
utilidad antes del REI & 2,3 & 2,2 & 4,9 & 1,8 \\
diferencia de cambio & & &
\end{tabular}




\begin{tabular}{lcccc} 
utilidad antes de impuestos & 147,9 & 239,6 & 213,4 & 195,3 \\
$\begin{array}{l}\text { participación de los } \\
\text { trabajadores }\end{array}$ & 15,9 & 26,0 & 0,0 & 0,0 \\
impuesto a la renta & 43,3 & 66,8 & 68,8 & 66,2 \\
\hline utilidad neta & 88,7 & 146,8 & 144,6 & 129,1 \\
\hline
\end{tabular}

Tabla 6: Estado de ganancias y pérdidas de la cadena de tiendas estudiada, años 2009; 2010; 2011 y 2012.

\section{CONCLUSIÓN}

La introducción de scanners en el conteo cíclico significó un ahorro en gastos de ventas de $\mathrm{S} / 1$ 886,50 mensualmente y de S/ 22 638,00 anualmente en los gastos de ventas en la sucursal estudiada (tienda ubicada en San Isidro).

Si se aplicase este proyecto en las 22 sucursales de esta cadena de tiendas, significaría un ahorro anual de S/ 205 800,00 , lo cual representa el $0,05 \%$ del total de gastos en la que incurre esta empresa para el año 2012.

No cabe duda que, en el entorno altamente cambiante en el cual se desarrollan todas las organizaciones, el avance tecnológico, variable no controlable, debe ser vista como una oportunidad en los planes estratégicos de toda empresa.

\section{BIBLIOGRAFÍA}

1. Agüero Zardón, L., Urquiola García, I. y Martínez Delgado, E. (2016). Propuesta de procedimiento para la gestión de inventarios. Técnica Administrativa, 15(2), 5-17. Recuperado de http://www.cyta.com.ar/ta1502/v15n2a2.htm

2. Esteves Pairazamán, A. T. y Fernández Bedoya, V. H. (2019). Aplicación de estrategias de ventas de tarjetas crediticias para incentivar al comercio electrónico en los clientes de una cadena de bancos en Perú. Revista Científica de la UCSA, 6(1), 23-32. DOI: http://dx.doi.org/10.18004/ucsa/2409-8752/2019.006(01)023-032

3. Dubs de Moya, R. (2012). El proyecto factible: una modalidad de investigación. Sapiens. Revista universitaria de investigación, 3(2), 1-18. Recuperado de https://www.redalyc.org/pdf/410/41030203.pdf

4. Durán, Y. (2012). Administración de inventario: elemento clave para la optimización de las utilidades en las empresas. Visión gerencial, 11(1), 55-78. Recuperado de http://www.redalyc.org/pdf/4655/465545892008.pdf

5. Fernández Bedoya, V. H. (2018). Punto de equilibrio y su incidencia en las decisiones financieras de empresas editoras en Lima. QUIPUKAMAYOC, 26(52), 95-101. DOI: http://dx.doi.org/10.15381/quipu.v26i52.15507

6. Garrido Bayas, I. Y. y Cejas Martínez, M. (2017). La gestión de inventario como factor estratégico en la administración de empresas. Negotium, 13(37), 109-129. Recuperado de http://www.redalyc.org/pdf/782/78252811007.pdf

7. Glover, B y Bhatt, H. (2009). RFID Essentials (1ra ed.). Massachusetts: Estados Unidos de América.

8. Gutiérrez, V. y Vidal, J. C. (2008). Modelos de Gestión de Inventarios en Cadenas de Abastecimiento: Revisión de la Literatura. Revista Facultad de Ingeniería Universidad Antioquía, 43, 134-149. Recuperado de https://aprendeenlinea.udea.edu.co/revistas/index.php/ingenieria/article/view/18765

9. Olivos Aarón, S. y Penagos Vargas, J. P. (2013). Modelo de Gestión de Inventarios: Conteo Cíclico por Análisis ABC. Ingeniare, 8(14) (2013) 107-111. DOI: https://doi.org/10.18041/1909-2458/ingeniare.14.617

10. Sanca Tinta, M. D. (2011). Tipos de Investigación Científica. Revista de Actualización Clínica, 9(1), 621-624. Recuperado de http://www.revistasbolivianas.org.bo/pdf/raci/v12/v12_a11.pdf 